\title{
Outcomes and complications of cataract surgery in patients with chronic ocular graft-versus-host-disease-a multicenter, retrospective analysis
}

\author{
Uta Gehlsen ${ }^{1,2} \cdot$ Christiane Faust $^{1} \cdot$ Christiane Blecha $^{3} \cdot$ Tina Dietrich-Ntoukas $^{4} \cdot$ Philipp Eberwein $^{5,6}$. \\ Susanne Issleib ${ }^{5} \cdot$ Tobias Meyer-ter-Vehn $^{7} \cdot$ Regine Braun $^{3} \cdot$ Henrike Westekemper $^{8} \cdot$ Philipp Steven $^{1,2}$ (i)
}

Received: 17 September 2021 / Revised: 20 February 2022 / Accepted: 22 February 2022 / Published online: 7 March 2022

(c) The Author(s) 2022

\begin{abstract}
Purpose To evaluate the outcome of phacoemulsification in patients with chronic ocular Graft-versus-host disease (oGVHD) after allogeneic hematopoietic stem cell transplantation (aHSCT).

Methods Retrospective, observational multicenter study from 1507 oGVHD patients. From the patient files, data were collected including best-corrected visual acuity (BCVA), intraocular pressure (IOP), Schirmer's test $I$, tear film break-up time (TFBUT), corneal fluorescein staining score, postoperative complications, and pre- and post-operative topical therapy. Results Seventy-three patients underwent cataract surgery in 104 eyes. In $n=84$ eyes, the oGVHD NIH grade was documented; $12 \%(n=12)$ of analyzed eyes were staged oGVHD NIH grade $1,31 \%(n=32) \mathrm{NIH} 2$ and $39 \%(n=41) \mathrm{NIH} 3$. The mean BCVA improved in $82 \%$ of the eyes ( $n=86$ eyes). BCVA significantly increased from $0.7 \pm 0.5$ to $0.4 \pm 0.4 \operatorname{LogMAR}$ after surgery independent from oGVHD severity. The mean IOP decreased from $14 \pm 4$ to $13 \pm 4 \mathrm{mmHg}$ after surgery. Visual acuity was moderately correlated to the pre-operative degree of corneal staining (Pearson $p=0.26, p=0.002$, Cohen's effect size $f=0.29)$. The visual acuity decreased by $0.078 \mathrm{LogMar}$ units $(95 \% \mathrm{CI}=0.027-0.141)$ with each increase of corneal staining by one grade $(p=0.05)$. After surgery, corneal epitheliopathy increased significantly in $42 \%(n=44)$ of the eyes. Postoperative complications included corneal perforation $(n=6,6 \%)$, cystoid macular edema $(n=4,4 \%)$, and endophthalmitis $(n=1,1 \%)$.

Conclusion Phacoemulsification in patients with chronic oGVHD significantly improves visual acuity, but is associated with an increased risk of complications in particular corneal epitheliopathy and corneal perforations.
\end{abstract}

Keywords Graft-versus-host-disease $\cdot$ Cataract $\cdot$ Phacoemulsification $\cdot$ Complication

Philipp Steven

philipp.steven@uk-koeln.de

1 Division for Dry-Eye and Ocular GvHD, Department of Ophthalmology, Medical Faculty, University and University Hospital of Cologne, Kerpener Strasse 62, 50937 Cologne, Germany

2 Cluster of Excellence: Cellular Stress Responses in Ageing-Associated Diseases, CECAD, University of Cologne, Cologne, Germany

3 Department of Ophthalmology, University Hospital Regensburg, Regensburg, Germany
4 Department of Ophthalmology, Charité Universitaetsmedizin Berlin, Corporate Member of Freie Universitaet Berlin, Humboldt Universitaet Berlin, and Berlin Institute of Health, Berlin, Germany

5 Eye Center, University Medical Center Freiburg, Freiburg, Germany

6 AugenCentrum Rosenheim, Rosenheim, Germany

7 University Eye Hospital, University Wuerzburg, Wuerzburg, Germany

8 Department of Ophthalmology, University Hospital Essen, University Duisburg Essen, Essen, Germany 


\section{Key Messages:}

- Phacoemulsification in patients with chronic ocular GVHD is described with high-success and very low complication rates, however clinically, severe complications and limited visual recovery are reported frequently.

- This retrospective multi-center study demonstrates, that although visual acuity increases in over $80 \%$, corneal epithelio pathy increases in over $40 \%$ of the patients and corneal perforation may even occur.

- Patients suffering from severe chronic ocular GVHD achieve lower visual acuity than those with mild-moderate ocular GVHD, despite the use of serum eye drops.

- The results implicate, that careful peri-operative care should be applied, to assure best possible visual rehabilitation and to avoid complications.

\section{Introduction}

Chronic graft-versus-host-disease (GVHD) is a major cause of morbidity in patients after allogeneic hematopoietic stem cell transplantation (aHSCT) as treatment for several hematological disorders such as acute lymphoblastic or myeloid leukemia. Acute or chronic ocular GvHD (oGVHD) is described in up to $60 \%$ of the patients, mainly affecting the ocular surface and adnexa [1-4]. Resulting in disturbed tear production [5], loss of meibomian glands [6-8], conjunctival scarring [9], corneal epithelial defects and perforation [10], corneal and conjunctival ulceration $[11,12]$, inflammation as well as consecutive ocular discomfort, pain and blurred vision, oGVHD leads to a significant reduction in the vision-related quality of life in these patients $[13,14]$. As increasing numbers of patients are receiving aHSCT and transplantation techniques and conditioning strategies have improved, patients' survival rates also increase [15]. Consequently, the number of oGVHD patients also increases with a consecutive increase of longterm complications.

One of the most common non-GVHD ocular pathologies following aHSCT is the formation of mainly subcapsular cataracts. Cataracts are caused by exposure to total body irradiation and/or chemotherapy, high-dose systemic corticosteroids, and also the prolonged use of topical or highdose systemic corticosteroids for prevention or treatment of systemic acute or chronic GvHD [16-18]. Due to significant visual impairment related to cataracts, many patients subsequently have to undergo phacoemulsification.

Generally, cataract surgery is a safe and effective procedure to restore visual acuity with very low complication rates $[19,20]$. In oGVHD patients, phacoemulsification is also considered safe without increased likelihood of severe complications [21-25], although post-operative findings are described such as aggravation of ocular surface disease including filamentary keratitis, corneal ulceration, and perforation. Other, presumably non-oGVHD related postoperative complications are cystoid macular edema (CME) and posterior capsular opacification [14, 22]. Publications on outcome and complications of phacoemulsification in oGVHD derive from single-center patient cohorts with low to moderate numbers $(n=7-41$ patients [21, 22, $25]$ ), and one larger study including $n=229$ patients with $n=51$ eyes undergoing cataract surgery [24]. Additionally, most studies published did not differentiate between severity of oGVHD. Shah et al. [23] evaluated the outcome in 10 eyes from 6 patients with severe GvHD and Saboo et al. [24] referred to two eyes treated with autologous serum eye drops as an indicator of severe ocular surface disease with distinct postoperative complications after cataract surgery. This published data, however, is often not reflected in daily clinical practice. Here, often patients with oGVHD that undergo cataract surgery present with severe worsening of the ocular surface and implicate a study with higher number of patients. Furthermore, neither the outcome of larger numbers of patients with severe oGVHD has been evaluated nor the outcome of phacoemulsification under the use of autologous serum tears.

The aim of this study was therefore to comparatively analyze data from 7 centers regarding the outcome after cataract surgery including complication rates in a larger number of patients, with a special focus on patients with severe oGvHD and application of autologous serum eye drops.

\section{Methods}

\section{Patient demographics}

For this retrospective, observational clinical study data from $n=1507$ ocular GvHD patients from 7 centers were identified in local electronic medical softwares such as ORBIS® (Agfa HealthCare, Bonn, Germany) by using ICD codes (H58.21-3). Anonymized data from 104 eyes from 73 consecutive patients ( $n=49$ male, $n=24$ female; average age $58 \pm 11$ years; range: $20-78$ years) undergoing 
phacoemulsification between $05 / 2010$ and $06 / 2018$ were manually extracted and then submitted as Excel ${ }^{\circledR}$ files by the participating centers for further analysis. Thirty-five patients had bilateral surgery (48\%). Cataract surgery was performed at a median interval of $3.4 \pm 2.5$ years (IQR: $2.3-4.8$ years) after allogeneic hematopoietic stem cell transplantation (aHSCT).

Underlying hematological diseases, concomitant ophthalmological diseases and treatments are depicted in Supplemental Tables 1, 2, 3 .

Best-corrected visual acuity (BCVA), intraocular pressure (IOP), Schirmer test $I$ (without anesthesia), tear film breakup time (TFBUT), and corneal fluorescein staining score (Oxford Scale[26]) were acquired during clinical routine examinations before and between 7 days up to one year after surgery. The median duration of the follow-up examination was $64 \pm 159 \mathrm{~d}$ (IQR: $28-145$ days) after cataract surgery.

In this study, $n=53(51 \%)$ of the patients were treated with undiluted autologous serum eye drops (ASED) produced in a sealed, sterile manufacturing system as described previously [27] or treated with albumin eye drops. In Germany, the application and coverage through public health insurance of ASED is restricted to most severely affected patients, based on decision by the Federal Constitutional Court in 2005. To qualify for cost-compensation and application of ASED, patients need to be threatened by "acute blindness" caused by corneal epitheliopathy. Although not evaluated in this survey, most patients that applied ASED (or albumin) were very likely to have been severely impacted by oGVHD before the first application of ASED.
However, patients with severe corneal epitheliopathy not always received ASED, which may also depends on absent availabilities of this therapy, refusal of cost-compensation, inability to donate blood, etc. For further analysis, data were grouped in autologous serum (ASED; $n=43$ )/albumin eye drops (AED; $n=10$ )-treated eyes versus no ASED/AEDtreated eyes. As inclusion criteria for further subgroup analysis regarding the impact of serum treatment, only eyes treated before and after surgery were included in the ASED/ AED group $(n=38 / 8)$; eyes without ASED/AED were used as control group $(n=46)$ (Table 1). For further sub-analysis, eyes were grouped regarding their GvHD NIH severity grade (NIH 0-3) according to [2].

\section{Statistical analysis}

For statistical analysis, data were tested for their normal distribution using Kolmogorov-Smirnov-test. Data were then analyzed using the non-parametric Wilcoxon signedrank test for paired samples to compare data before and after surgery (SPSS software, version 25, IBM, Ehningen, Germany). P-values $<0.05$ were considered to be significant. For comparison between ASED and NIH grade related groups Mann-Whitney-U Test or Kruskal Wallis test followed by a Bonferroni corrected Dunn's post hoc analysis was used. All ophthalmological data are depicted as boxplot diagrams, demonstrating the median with $25 \%$ and $75 \%$ percentiles (box) as well as the highest and lowest values (whiskers). Outliers are depicted as circles. For correlation of visual
Table 1 Number of patients treated with autologous serum (ASED) or albumin eye drops (AED) before and after surgery in total and $\mathrm{NIH} \mathrm{1,2,} \mathrm{and} 3$ group

\begin{tabular}{llll}
\hline & $\begin{array}{l}\text { NIH1 } \\
\text { Number of patients }(n=) / \\
\text { percentage }\end{array}$ & $\begin{array}{l}\text { NIH2 } \\
\text { Number of patients }(n=) / \\
\text { percentage }\end{array}$ & $\begin{array}{l}\text { NIH3 } \\
\text { pumber of } \\
\text { percents }(n=) /\end{array}$ \\
\hline noASED/AED & $10 / 91 \%$ & $21 / 58 \%$ & $15 / 33 \%$ \\
ASED & $1 / 9 \%$ & $9 / 25 \%$ & $28 / 62 \%$ \\
AED & $0 / 0 \%$ & $6 / 17 \%$ & $2 / 4 \%$ \\
Total number of patients & 11 & 36 & 45 \\
\hline
\end{tabular}

and tear film break-up-time were not influenced. Data were presented as mean/median \pm standard deviation (minimum-maximum value)
Table 2 Ophthalmological parameters of 104 eyes included in the analysis. After surgery visual acuity and intraocular pressure improved, but corneal staining exacerbated significantly. Schirmer's

\begin{tabular}{llll}
\hline & $\begin{array}{l}\text { Before surgery } \\
\text { mean/median } \pm \text { standard deviation (range) }\end{array}$ & $\begin{array}{l}\text { After surgery } \\
\text { mean/median } \pm \text { standard deviation (range) }\end{array}$ & $\begin{array}{l}\text { Significance } \\
\text { BCVA (LogMAR) }\end{array}$ \\
IOP (mmHg) & $0.7 / 0.6 \pm 0.5(0.1-2.0)$ & $0.4 / 0.2 \pm 0.4(0-2.0)$ & $p=0.0001$ \\
Staining (Oxford) & $14 / 15 \pm 4(5-25)$ & $13 / 12 \pm 4(5-23)$ & $p=0.0001$ \\
Schirmer's $I$ (mm) & $2.1 / 2 \pm 1.4(0-5)$ & $2.5 / 2 \pm 1.7(0-5)$ & $p=0.009$ \\
TFBUT (s) & $3 / 2 \pm 6(0-35)$ & $4 / 1 \pm 7(0-35)$ & $p=0.6$ \\
\hline
\end{tabular}


outcome and ophthalmological parameters, the Spearman coefficient and a linear regression were calculated.

\section{Results}

\section{Ophthalmological parameters}

The mean best-corrected visual acuity (BCVA) in this study cohort improved significantly after surgery. The mean corneal staining significantly increased after surgery from 2.1 to 2.5 (Table 1). The mean intraocular pressure (IOP) decreased; Schirmer's $I$ value and tear film breakup time (TFBUT) were not influenced by cataract surgery. All parameters are described in detail in Table 2 (mean/ median \pm standard deviation (range: $\min -\max )$ ).

\section{Impact of oGVHD severity on outcome of surgery}

\section{Visual function, intraocular pressure, tear film}

Ocular GvHD severity before surgery was graded using NIH consensus criteria [2, 28, 29]. Twelve percent of the patients $(n=12$ eyes) were classified as NIH grade $1,31 \%(n=32$ eyes) NIH grade 2 , and $39 \%$ ( $n=41$ eyes) were NIH grade 3 . For $18 \%$ of the patients ( $n=19$ eyes), no information concerning the NIH grading was obtainable.

As a weak correlation between the NIH grade and the visual outcome after surgery was observed (Spearman-Rho coefficient: $\rho=0.2, p=0.05$ ), eyes were assigned to different groups according to the NIH grade and were compared regarding their visual outcome. The BCVA improved in all NIH groups ( $p=0.0001)$, independent of the NIH grading: mean BVCA LogMAR before surgery was NIH 1: $0.6 \pm 0.4$,
NIH 2: $0.7 \pm 0.5$, NIH 3: $0.8 \pm 0.4$ and mean BVCA LogMAR after surgery was NIH 1: $0.2 \pm 0.4(p=0.005)$, NIH 2: $0.4 \pm 0.5(p<0.001)$, NIH 3: $0.4 \pm 0.4(p<0.001)$ (Fig. 1B). The overall success rate was $82 \%$, with success defined as improvement of visual acuity after surgery $(n=85$ eyes out of 104 eyes improved). In the NIH 1 group, success rate was 92\% ( $n=11$ out of 12 eyes); in NIH $278 \%$ ( $n=25$ out of 32 eyes), and in NIH $382 \%$ ( $n=34$ out of 41 eyes). In eyes without NIH grade, available success rate was $79 \%(n=15$ out of 19 eyes).

All NIH groups demonstrated comparable values in Schirmer's test and TFBUT before and after surgery although the TFBUT in NIH 3 was decreased compared to NIH 2 after surgery $(p=0.003)$. As expected after cataract surgery, the mean IOP values decreased in all eyes after surgery: $\mathrm{NIH} 1(15 \pm 4 / 13 \pm 5 \mathrm{mmHG}), \mathrm{NIH} 2(15 \pm 3 / 13 \pm 4 \mathrm{mmHg}$, $p=0.005)$, and NIH $3(14 \pm 4 / 12 \pm 4 \mathrm{mmHg}, p=0.001)$. Detailed ophthalmological data are listed in Supplemental Table 4.

\section{Corneal epitheliopathy}

Since the postoperative visual outcome was correlated with the corneal staining before surgery (Spearman-Rho coefficient $\rho=0.5, p=0.0001$ ), a linear regression was calculated. The visual acuity was moderately correlated to the pre-operative degree of corneal staining (Pearson $r=0.26, p=0.002$, Cohen's effect size $f=0.29)$. The visual acuity decreased by $0.078 \mathrm{LogMar}$ units $(95 \% \mathrm{CI}=0.027-0.141)$ with each increase of corneal staining by one grade $(p=0.05)$ (Supplemental Fig. 1). Due to the characteristics of the NIH criteria, a significant difference between the NIH groups existed before surgery $(p=0.003)$, with higher staining score in $\mathrm{NIH} 3$ (mean \pm SD: $2.8 \pm 1.6)$ compared to NIH $1(1.3 \pm 0.7$;
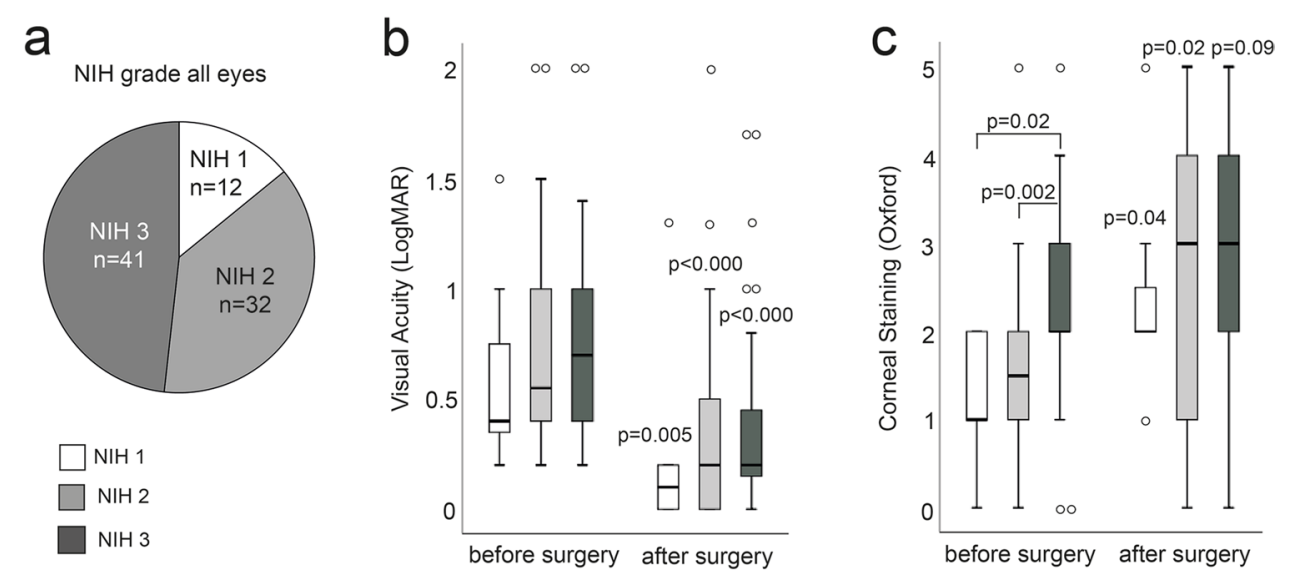

Fig. 1 Outcome after cataract surgery related to severity of oGVHD (NIH grade 1-3). a NIH grade of $n=84$ oGVHD eyes (in 20 eyes $\mathrm{NIH}$ grades were not documented). Twelve percent of analyzed eyes were graded NIH 1, 31\% NIH 2, and 39\% were NIH 3. b Visual acu- ity after cataract surgery improved in all groups independent from the NIH grade. c In NIH 1 and 2 staining increased after surgery to an NIH 3 level. NIH 3 group had a significantly higher corneal fluorescein staining at baseline compared to NIH 1 and 2 
Fig. 2 Corneal epitheliopathy after cataract surgery in $n=76$ eyes calculated from the alteration in corneal staining (Oxford) before and after surgery. In $54 \%$ of the eyes, staining was impaired after surgery; in 23\% of the eyes, corneal staining did not change. In $16 \%$, corneal staining improved. In NIH 1 group, $63 \%$ of the eyes impaired, in NIH $265 \%$, and in NIH $345 \%$

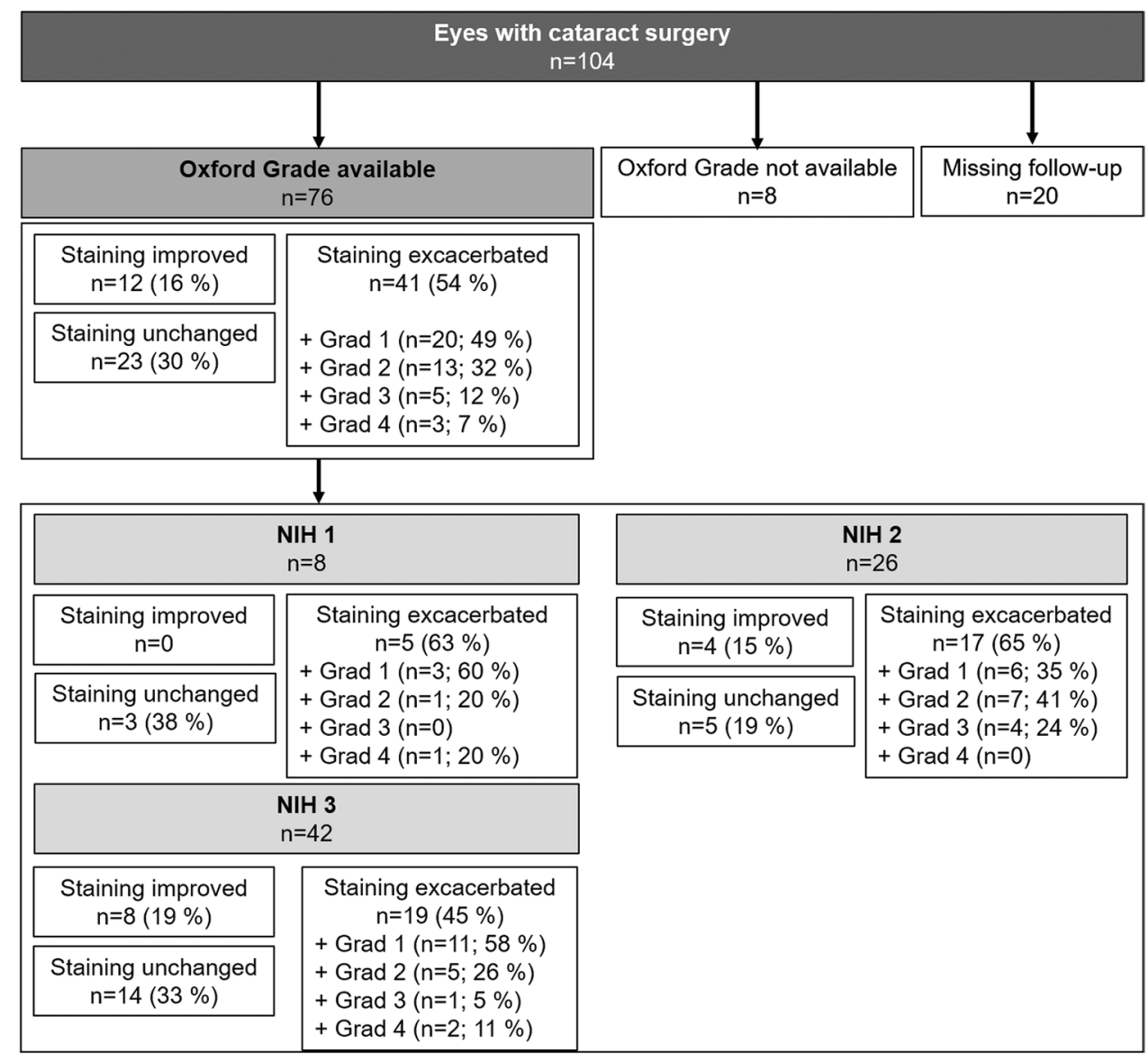

$p=0.02)$ and NIH $2(1.8 \pm 0.4 ; p=0.002)$. Detailed data are listed in Supplemental Table 4. After surgery, the staining increased significantly in NIH $2(3.2 \pm 0.4, p=0.02)$ and $\mathrm{NIH} 1(2.4 \pm 1.2, p=0.04)$ and reached a comparable degree of staining as in the NIH $3(2.8 \pm 1.6)$ group. The average staining in the NIH 3 group did not change after surgery (Fig. 1C).
Besides the increase of average staining after the procedure, exacerbation of corneal staining was documented in $46 \%(n=48)$ of all 104 eyes investigated. The Oxford staining score was available in $n=76$ eyes, in $n=8$ eyes staining was not documented using the Oxford grading scale, and $n=20$ data points were missing after surgery. In $49 \%(n=20$ eyes) out of these, the eyes worsened by 1 Oxford grade, $32 \%(n=13)$ by 2 grades, $12 \%(n=5)$ by 3 grades, and $7 \%$
Table 3 Ophthalmological parameters before and outcome after cataract surgery in ASED/ AED treated eyes compared to control eyes without ASED/ AED

\begin{tabular}{|c|c|c|c|c|}
\hline & \multicolumn{2}{|c|}{ ASED/AED } & \multirow{2}{*}{$\begin{array}{l}\text { No ASED/AED } \\
0.7 / 0.5 \pm 0.4(0.1-2)\end{array}$} & \multirow{2}{*}{$\begin{array}{l}\begin{array}{l}\text { Significance } \\
\text { (ASED/ } \\
\text { AED vs. } \\
\text { control) }\end{array} \\
p=0.01\end{array}$} \\
\hline BCVA (LogMAR) & pre & $0.8 / 0.7 \pm 0.5(0.2-2)$ & & \\
\hline & post & $0.4 / 0.2 \pm 0.4(0-2)$ & $0.3 / 0.2 \pm 0.4(0-1.7)$ & $p=0.05$ \\
\hline \multirow{2}{*}{ IOP (mmHg) } & pre & $14 / 14 \pm 4(5-20)$ & $15 / 15 \pm 4(7-25)$ & $p=0.7$ \\
\hline & post & $12 / 12 \pm 4(5-23)$ & $13 / 13 \pm 4(6-22)$ & $p=0.3$ \\
\hline \multirow[t]{2}{*}{ Staining (Oxford) } & pre & $2.6 / 3 \pm 1.2(1-5)$ & $1.9 / 2 \pm 1.4(0-5)$ & $p=0.02$ \\
\hline & post & $3.1 / 3 \pm 1.4(1-5)$ & $2.3 / 2 \pm 2.7(0-5)$ & $p=0.06$ \\
\hline \multirow[t]{2}{*}{ Schirmer's I (mm) } & pre & $2 / 3 \pm 3(0-10)$ & $4 / 1 \pm 7(0-35)$ & $p=0.9$ \\
\hline & post & $2 / 0 \pm 3(0-10)$ & $6 / 3 \pm 9(0-35)$ & $p=0.4$ \\
\hline \multirow[t]{2}{*}{ TFBUT (s) } & pre & $2 / 1 \pm 2(0-8)$ & $3 / 2 \pm 2(0-8)$ & $p=0.3$ \\
\hline & post & $2 / 1 \pm 2(0-8)$ & $3 / 2 \pm 2(0-8)$ & $p=0.2$ \\
\hline
\end{tabular}


$(n=3)$ by 4 grades. In $30 \%(n=23)$ of all eyes, no change was documented; in $16 \%(n=12)$ of the eyes, staining score improved after surgery (Fig. 2). The post-operative alteration of corneal staining did not correlate with NIH grade; in the NIH 1 group, $63 \%(n=5)$ of the eyes worsened, in NIH 2 graded eyes $65 \%(n=17)$, and in NIH 3 graded eyes $45 \%$ $(n=19)$ (Fig. 2).

\section{ASED/AED treatment}

The eyes were grouped in ASED/AED treated $(n=46)$ or control eyes (without ASED/AED treatment; $n=46$ ) to analyze the impact of serum treatment. In both groups, visual acuity improved significantly after surgery $(p=0.0001$ each). However, in eyes treated with ASED/AED, a significantly worse visual acuity was observed before and after surgery compared to control eyes (Table 2). ASED/ AED eyes showed significantly higher staining before surgery compared to control eyes. After surgery, staining further increased in control eyes $(p=0.009)$ and by trend in ASED/AED eyes $(p=0.08)$ compared to pre-operative findings. After surgery staining in ASED/AED, the eyes also increased by trend $(p=0.06)$ compared to control eyes (Table 3). ASED/AED treatment did not affect IOP, TFBUT, or Schirmer's in this study.

Out of the NIH 1 group, 9\% $(n=1)$ of the patients were treated with topical ASED or AED, $42 \%$ out of the NIH 2 ( $n=9$ ASED, $n=6$ AED), but significantly more from the NIH 3 group (68\%, $n=28$ ASED, $n=2$ AED) compared to NIH 2 (Table 1). To further investigate the influence of serum (ASED/AED) treatment on the outcome in severe cases of oGVHD, a sub-analysis of NIH 3 eyes was performed and ASED-treated (before and after surgery) eyes were compared with eyes without ASED (Fig. 3, Supplemental Table 5).

\section{Complications}

Increase in corneal staining was documented as the most common side effect after cataract surgery in 42 eyes $(40 \%$ of all patients). Overall, 15 eyes, (14\% of all patients) developed further complications after cataract surgery (Table 4). The second most frequent complication was corneal perforation in 6 eyes ( $6 \%$ of all eyes) and macular edema (CME) in 4 eyes ( $4 \%$ of all eyes). One patient developed (bacterial) endophthalmitis (1\%). This patient later died due to severe complications of systemic GvHD.

Out of the $n=53$ eyes with documented complications, $11 \%$ were NIH $1(n=6), 37 \%$ were NIH $2(n=20)$, and $36 \%$ NIH $3(n=19)$. In $n=8$ eyes with complications, no NIH documentation was available.

In patients with ASED/AED treatment complications were documented in 56\% $(n=26)$, and in patients without
ASED/AED, 78\% ( $n=36)$ of the eyes developed complications. In 3 patients, a prolonged increased corneal staining occurred despite ASED treatment after cataract surgery. One patient reported worsening of epitheliopathy after discontinuation of ASED treatment. In severe NIH 3 graded eyes, the overall complication rate was $44 \%(n=12)$ in the ASED/ AED group, and $47 \%(n=7)$ in the control group without ASED/AED. The number of severe complications in all eyes was decreased by trend $(p=0.09)$ in the ASED/AED group $(6 \%, n=1)$ compared to eyes without ASED/AED $(27 \%$, $n=9$ ). In the NIH 3 subgroup, no severe complications were documented in the ASED/AED group, but two out of 7 eyes of the control group had severe complications after surgery. A statistical analysis was not applicable due to low numbers in the subgroups.

\section{Discussion}

In concordance to other studies, postoperative visual improvement after phacoemulsification in ocular GVHD as the main outcome measure in this retrospective study was significant, resulting in a success rate of $82 \%$. This is slightly lower than published outcomes of $87-97 \%$ [21, 23, 24] and possibly due to a larger proportion of cases with severe ocular GVHD in our cohort. In this context, the enhancement of visual acuity was correlated to the pre-operative degree of corneal staining but independent of the severity of oGVHD. A possible cause might be, that differences in NIH grading and in the availability of topical serum/albumin therapy in the contributing centers influenced the data or activity of ocular surface inflammation was higher than anticipated. It should be considered that sub-clinical ocular surface inflammation may have contributed to poor visual outcomes as discussed in [22]. Therefore, a revised grading scheme is warranted that enables better differentiation of oGVHD severity and activity in the future [30, 31].

It is well known that cataract surgery can induce or aggravate pre-existing dry eye disease, specifically corneal complications such as recurrent epithelial defects, filamentary, or punctate keratitis [32-34]. In this study, epitheliopathy aggravated in $42 \%$ and corneal staining increased significantly in the overall population investigated, independent from NIH grade. This increase could be due to desiccation during the procedure, epithelial toxicity of the disinfecting agents applied to the ocular surface, extend of the incisions, experience of the surgeon, time of the surgery and other factors that should be evaluated in a prospective followup study. Previously, the incidence of epithelial defects after cataract surgery in oGVHD was reported much lower between 0 and $8 \%$ [21, 24, 25]. Also, a temporary worsening of epithelial damages in 16\% was reported 1-4 weeks after surgery [24], but the staining decreased postoperatively to 

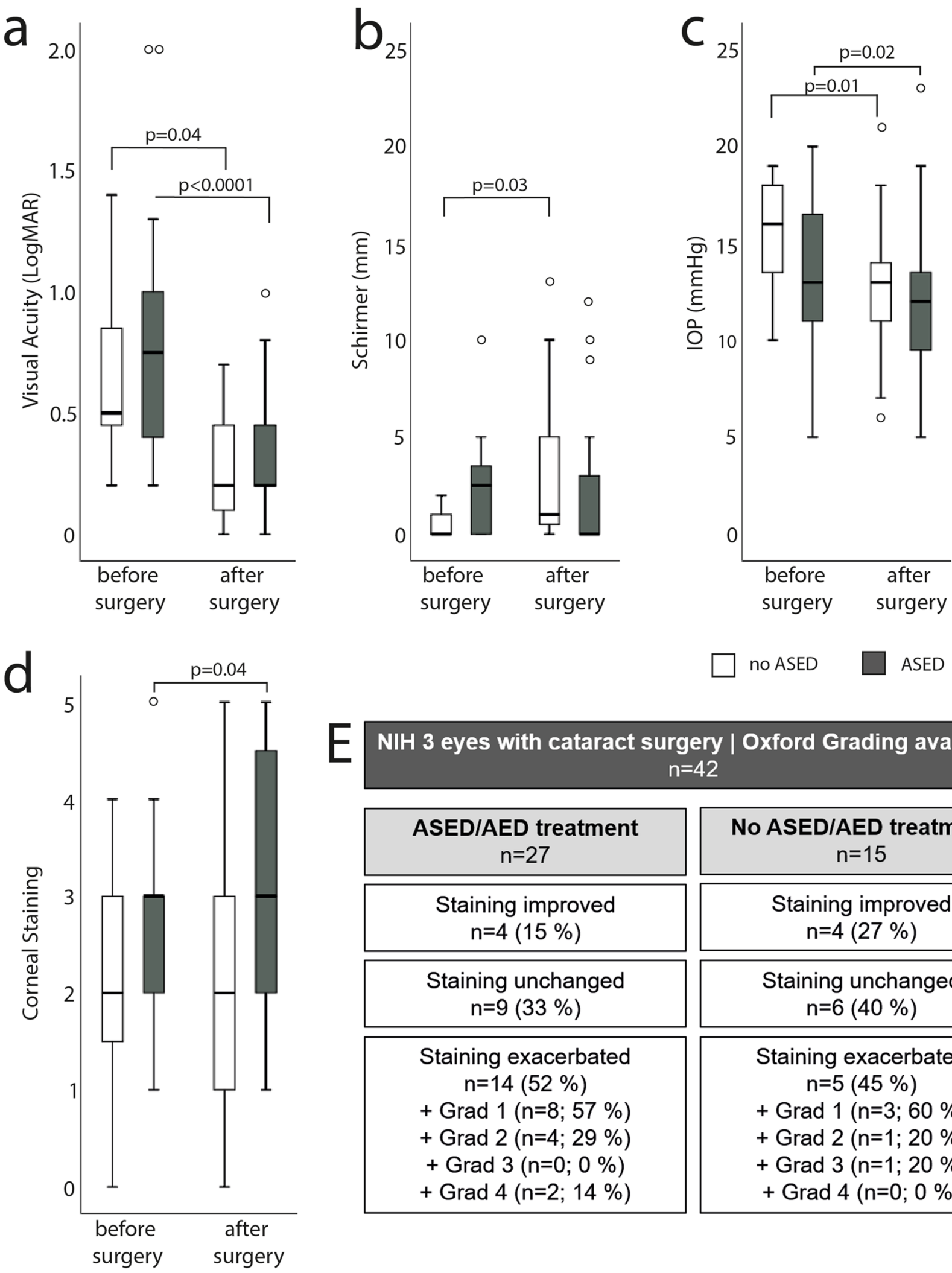

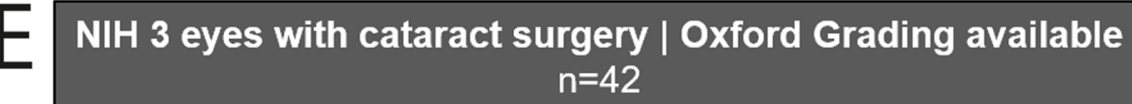

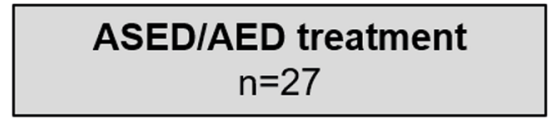

Staining improved
$\mathrm{n}=4(15 \%)$

Staining unchanged $n=9(33 \%)$

Staining exacerbated $n=14(52 \%)$

+ Grad $1(n=8 ; 57 \%)$

+ Grad 2 (n=4; $29 \%)$

+ Grad $3(n=0 ; 0 \%)$

+ Grad $4(n=2 ; 14 \%)$

\section{No ASED/AED treatment} $n=15$

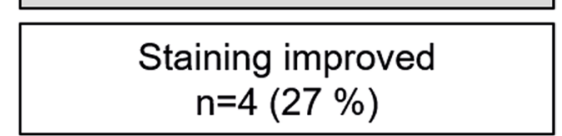
$n=4(27 \%)$

Staining unchanged $n=6(40 \%)$

Staining exacerbated $n=5(45 \%)$

+ Grad $1(n=3 ; 60 \%)$

+ Grad $2(n=1 ; 20 \%)$

+ Grad $3(n=1 ; 20 \%)$

+ Grad $4(n=0 ; 0 \%)$
Fig. 3 Ophthalmological parameters prior and after cataract surgery in $n=27$ ASED-treated and $n=15$ control eyes (no ASED) with NIH grade 3 only. a Visual acuity improved in both groups. b Schirmer's increased in control eyes, but not in ASED-treated eyes. $\mathbf{c}$ Intraocular pressure decreased in both groups. $\mathbf{d}$ Mean corneal staining increased in ASED-treated eyes after surgery. e Changes in corneal epitheliopathy after surgery in ASED and control eyes. No statistical differences between both treatment groups were observed in the number of eyes exacerbating after surgery. (ASED = autologous serum eye drops) 
Table 4 Cataract surgery-related post-operative complications. Overall $n=53$ eyes (from 104 eyes overall) with complications were documented

\begin{tabular}{ll}
\hline Post-surgery findings/complications & $n=(\%$ of all eyes $)$ \\
\hline Increased corneal staining & $44(42 \%)$ \\
Corneal perforation & $6(6 \%)$ \\
Cystoid macular edema (CME) & $4(4 \%)$ \\
Corneal cloudiness & $2(2 \%)$ \\
Increase intraocular pressure & $1(1 \%)$ \\
Endophthalmitis & $1(1 \%)$ \\
\hline
\end{tabular}

a pre-surgery level after that time, comparable to patients without oGVHD or dry eye disease after cataract surgery [35]. However, in this study, corneal staining was still increased at up to 5 months after phacoemulsification. Six patients developed corneal erosions and five of these progressed to corneal perforations. Filamentary keratitis was present only in one patient but already diagnosed before surgery, which represents a lower rate than reported before [24].

Although controversially discussed, in our experience, ASED treatment using a sealed, sterile manufacturing system is an efficient and safe treatment option to improve visual outcome and ocular surface in chronic oGVHD [27]. Thirty-seven percent of the patients in this study were treated with $100 \%$ ASED, and an additional $8 \%$ with AED prior and after cataract surgery, however, that did not prevent increase of staining after phacoemulsification. An explanation could be, that ASED treatment was clinically only applied in severe cases, that otherwise would not have been suitable for cataract surgery, and that might have had a higher risk of corneal complications in general. However, although presenting with increased corneal staining, ASED-treated eyes demonstrated a lower rate of severe complications such as perforations. These findings support the strategy to pre-treat severe patients with serum eye drops to achieve a higher degree of peri-operative safety.

Elevated IOP is another general complication in oGVHD, due to the frequent use of steroids and corneal scarring [36]. Balaram et al. [21] reported elevated IOP after cataract surgery in three eyes as an early postoperative complication. In this study, intraocular pressure decreased slightly after surgery in $87 \%$ of the patients. However, the majority of the patients had an IOP $\leq 20 \mathrm{mmHg}$ before and after surgery, which is within the normal range. Further severe complications in this study were CME in $4 \%$, which was comparable to $4-5.6 \%$ as reported in $[22,24]$. Posterior capsular opacification (PCO) requiring YAG capsulometry was documented in $2 \%$, representing a distinct lower rate than published in [21] (62\% PCO; 44\% YAG) and [24] (18\% PCO). However, the rate is likely to be much higher since the procedure is mainly performed in private practice and was therefore maybe not documented in the according files of the participating academic centers.

From our experience, phacoemulsification in oGVHD should be restricted to experienced surgeons. If cataract occurs following aHSCT, earlier rather than later surgery with respect to progression of oGVHD should also be considered in order to achieve better postoperative visual outcomes. However, in cases of highly active oGVHD associated with severe inflammatory activity on the ocular surface, cataract surgery may be postponed. If severe corneal epitheliopathy is present and surgery necessary, pre-operative application of autologous/allogeneic serum eye drops in addition to topical cyclosporine seems favorable. From these findings, the question arises, whether there is perioperative management that would further reduce complications and improve surgical outcome. Most participating centers now recommend a pre-operative application of topical corticosteroids (e.g. unpreserved dexamethasone TID for 3 consecutive days before surgery) followed by weekly examinations and aggressive therapy of ocular surface alterations as a standard. Overall, peri-operative care is crucial in oGVHD and external eye care providers should be informed about the increased complication risks in this special group of patients.

In summary, the frequent worsening of ocular surface disease after cataract surgery found in this study as a result of underlying oGVHD and/or as a consequence of the surgical procedure can cause severe complications which need to be monitored and treated. Future studies are warranted to establish guidelines on phacoemulsification in oGVHD, to enhance surgical outcome while reducing complications.

Supplementary Information The online version contains supplementary material available at https://doi.org/10.1007/s00417-022-05613-w.

Funding Open Access funding enabled and organized by Projekt DEAL. This study is funded by the Division of Dry-eye and ocular GVHD received donations from Novaliq, Ursapharm, and Juergen and Monika Ziehm.

\section{Declarations}

Ethics approval All procedures performed in studies involving human participants were in accordance with the ethical standards of the local ethics committees and with the 1964 Helsinki declaration and its later amendments or comparable ethical standards. Ethical approval was obtained by the local ethics committee approval number: $20-1141$ in view of the retrospective nature of the study and all procedures being performed were part of the routine care.

Conflict of interest P.S. has received financial support from Novaliq $\mathrm{GmbH}$, Roche, Bausch\&Lomb, and Ursapharm. All other authors declare no competing interests. 
Open Access This article is licensed under a Creative Commons Attribution 4.0 International License, which permits use, sharing, adaptation, distribution and reproduction in any medium or format, as long as you give appropriate credit to the original author(s) and the source, provide a link to the Creative Commons licence, and indicate if changes were made. The images or other third party material in this article are included in the article's Creative Commons licence, unless indicated otherwise in a credit line to the material. If material is not included in the article's Creative Commons licence and your intended use is not permitted by statutory regulation or exceeds the permitted use, you will need to obtain permission directly from the copyright holder. To view a copy of this licence, visit http://creativecommons.org/licenses/by/4.0/.

\section{References}

1. Hessen M, Akpek EK (2012) Ocular graft-versus-host disease. Curr Opin Allergy Clin Immunol 12:540-547. https://doi.org/10. 1097/ACI.0b013e328357b4b9

2. Filipovich AH, Weisdorf D, Pavletic S, Socie G, Wingard JR, Lee SJ, Martin P, Chien J, Przepiorka D, Couriel D, Cowen EW, Dinndorf P, Farrell A, Hartzman R, Henslee-Downey J, Jacobsohn D, McDonald G, Mittleman B, Rizzo JD, Robinson M, Schubert M, Schultz K, Shulman H, Turner M, Vogelsang G, Flowers ME (2005) National Institutes of Health consensus development project on criteria for clinical trials in chronic graft-versus-host disease: I. Diagnosis and staging working group report. Biol Blood Marrow Transplant 11:945-956. https://doi.org/10.1016/j.bbmt. 2005.09.004

3. Ferrara JL, Levine JE, Reddy P, Holler E (2009) Graft-versus-host disease. Lancet 373:1550-1561. https://doi.org/10.1016/S01406736(09)60237-3

4. Giannaccare G, Pellegrini M, Bernabei F, Scorcia V, Campos E (2019) Ocular surface system alterations in ocular graft-versushost disease: all the pieces of the complex puzzle. Graefes Arch Clin Exp Ophthalmol 257:1341-1351. https://doi.org/10.1007/ s00417-019-04301-6

5. Na KS, Yoo YS, Hwang KY, Mok JW, Joo CK (2015) Tear osmolarity and ocular surface parameters as diagnostic markers of ocular graft-versus-host disease. Am J Ophthalmol 160(143149):e141. https://doi.org/10.1016/j.ajo.2015.04.002

6. Ogawa Y, Okamoto S, Wakui M, Watanabe R, Yamada M, Yoshino M, Ono M, Yang HY, Mashima Y, Oguchi Y, Ikeda Y, Tsubota K (1999) Dry eye after haematopoietic stem cell transplantation. Br J Ophthalmol 83:1125-1130. https://doi.org/10. 1136/bjo.83.10.1125

7. Ban Y, Ogawa Y, Ibrahim OM, Tatematsu Y, Kamoi M, Uchino M, Yaguchi S, Dogru M, Tsubota K (2011) Morphologic evaluation of meibomian glands in chronic graft-versus-host disease using in vivo laser confocal microscopy. Mol Vis 17:2533-2543

8. Hwang HS, Ha M, Kim HS, Na KS (2019) Longitudinal analysis of meibomian gland dropout in patients with ocular graft-versushost disease. Ocul Surf 17:464-469. https://doi.org/10.1016/j.jtos. 2019.04.009

9. Kheirkhah A, Coco G, Satitpitakul V, Dana R (2018) Subtarsal fibrosis is associated with ocular surface epitheliopathy in graftversus-host disease. Am J Ophthalmol 189:102-110. https://doi. org/10.1016/j.ajo.2018.02.020

10. Tranos PG, Forbes J, Jagger J (2001) Corneal perforation in chronic graft-versus-host disease. Eye (Lond) 15:111-113. https://doi.org/10.1038/eye.2001.29

11. Sinha S, Singh RB, Dohlman TH, Taketani Y, Yin J, Dana R (2020) Prevalence and risk factors associated with corneal perforation in chronic ocular graft-versus-host-disease. Cornea. https://doi.org/10.1097/ICO.0000000000002526

12. Yeh PT, Hou YC, Lin WC, Wang IJ, Hu FR (2006) Recurrent corneal perforation and acute calcareous corneal degeneration in chronic graft-versus-host disease. J Formos Med Assoc 105:334-339. https://doi.org/10.1016/s0929-6646(09)60125-x

13. Riemens A, Te Boome LC, Kalinina Ayuso V, Kuiper JJ, Imhof SM, Lokhorst HM, Aniki R (2014) Impact of ocular graftversus-host disease on visual quality of life in patients after allogeneic stem cell transplantation: questionnaire study. Acta Ophthalmol 92:82-87. https://doi.org/10.1111/aos.12047

14. Saboo US, Amparo F, Abud TB, Schaumberg DA, Dana R (2015) Vision-related quality of life in patients with ocular graft-versus-host disease. Ophthalmology 122:1669-1674. https://doi.org/10.1016/j.ophtha.2015.04.011

15. Wolff D, Schleuning M, von Harsdorf S, Bacher U, Gerbitz A, Stadler M, Ayuk F, Kiani A, Schwerdtfeger R, Vogelsang GB, Kobbe G, Gramatzki M, Lawitschka A, Mohty M, Pavletic SZ, Greinix H, Holler E (2011) Consensus conference on clinical practice in chronic GVHD: second-line treatment of chronic graft-versus-host disease. Biol Blood Marrow Transplant 17:117. https://doi.org/10.1016/j.bbmt.2010.05.011

16. Belkacemi Y, Labopin M, Vernant JP, Prentice HG, Tichelli A, Schattenberg A, Boogaerts MA, Ernst P, Della Volpe A, Goldstone AH, Jouet JP, Verdonck LF, Locasciulli A, Rio B, Ozsahin M, Gorin NC (1998) Cataracts after total body irradiation and bone marrow transplantation in patients with acute leukemia in complete remission: a study of the European Group for Blood and Marrow Transplantation. Int J Radiat Oncol Biol Phys 41:659-668. https://doi.org/10.1016/s0360-3016(98)00077-7

17. van Kempen-Harteveld ML, Struikmans H, Kal HB, van der Tweel I, Mourits MP, Verdonck LF, Schipper J, Battermann JJ (2000) Cataract-free interval and severity of cataract after total body irradiation and bone marrow transplantation: influence of treatment parameters. Int J Radiat Oncol Biol Phys 48:807-815. https://doi.org/10.1016/s0360-3016(00)00669-6

18. Benyunes MC, Sullivan KM, Deeg HJ, Mori M, Meyer W, Fisher L, Bensinger R, Jack MK, Hicks J, Witherspoon R et al (1995) Cataracts after bone marrow transplantation: long-term follow-up of adults treated with fractionated total body irradiation. Int J Radiat Oncol Biol Phys 32:661-670. https://doi.org/ 10.1016/0360-3016(94)00392-x

19. Wang J, Su F, Wang Y, Chen Y, Chen Q, Li F (2019) Intra and post-operative complications observed with femtosecond laser-assisted cataract surgery versus conventional phacoemulsification surgery: a systematic review and meta-analysis. BMC Ophthalmol 19:177. https://doi.org/10.1186/s12886-019-1190-2

20. Manning S, Barry P, Henry Y, Rosen P, Stenevi U, Young D, Lundstrom M (2016) Femtosecond laser-assisted cataract surgery versus standard phacoemulsification cataract surgery: study from the European Registry of Quality Outcomes for Cataract and Refractive Surgery. J Cataract Refract Surg 42:1779-1790. https://doi.org/10.1016/j.jcrs.2016.10.013

21. Balaram M, Dana MR (2001) Phacoemulsification in patients after allogeneic bone marrow transplantation. Ophthalmology 108:1682-1687. https://doi.org/10.1016/s0161-6420(01) 00675-3

22. de Melo FR, Kron-Gray MM, De la Parra-Colin P, He Y, Musch DC, Mian SI, Niziol L, Soong HK (2015) Outcomes of cataract surgery in graft-versus-host disease. Cornea 34:506-511. https:// doi.org/10.1097/ICO.0000000000000395

23. Shah A, Santhiago MR, Espana EM (2016) Cataract surgery in patients with chronic severe graft-versus-host disease. J Cataract Refract Surg 42:833-839. https://doi.org/10.1016/j.jcrs.2016.02. 046 
24. Saboo US, Amparo F, Shikari H, Jurkunas UV, Dana R (2015) Outcomes of phacoemulsification in patients with chronic ocular graft-versus-host disease. Graefes Arch Clin Exp Ophthalmol 253:901-907. https://doi.org/10.1007/s00417-015-2940-3

25. Penn EA, Soong HK (2002) Cataract surgery in allogeneic bone marrow transplant recipients with graft-versus-host disease(1). J Cataract Refract Surg 28:417-420

26. Bron AJ, Evans VE, Smith JA (2003) Grading of corneal and conjunctival staining in the context of other dry eye tests. Cornea 22:640-650

27. Tahmaz V, Gehlsen U, Sauerbier L, Holtick U, Engel L, Radojska S, Petrescu-Jipa VM, Scheid C, Hallek M, Gathof B, Cursiefen C, Steven P (2017) Treatment of severe chronic ocular graft-versus-host disease using 100\% autologous serum eye drops from a sealed manufacturing system: a retrospective cohort study. $\mathrm{Br}$ J Ophthalmol 101:322-326. https://doi.org/10.1136/bjophthalm ol-2015-307666

28. Arai S, Jagasia M, Storer B, Chai X, Pidala J, Cutler C, Arora M, Weisdorf DJ, Flowers ME, Martin PJ, Palmer J, Jacobsohn D, Pavletic SZ, Vogelsang GB, Lee SJ (2011) Global and organ-specific chronic graft-versus-host disease severity according to the 2005 NIH Consensus Criteria. Blood 118:4242-4249. https://doi.org/ 10.1182/blood-2011-03-344390

29. Mitchell SA, Jacobsohn D, Thormann Powers KE, Carpenter PA, Flowers ME, Cowen EW, Schubert M, Turner ML, Lee SJ, Martin P, Bishop MR, Baird K, Bolanos-Meade J, Boyd K, Fall-Dickson JM, Gerber LH, Guadagnini JP, Imanguli M, Krumlauf MC, Lawley L, Li L, Reeve BB, Clayton JA, Vogelsang GB, Pavletic SZ (2011) A multicenter pilot evaluation of the National Institutes of Health chronic graft-versus-host disease (cGVHD) therapeutic response measures: feasibility, interrater reliability, and minimum detectable change. Biol Blood Marrow Transplant 17:1619-1629. https://doi.org/10.1016/j.bbmt.2011.04.002

30. Blecha C, Wolff D, Holler B, Holler E, Weber D, Vogt R, Helbig H, Dietrich-Ntoukas T (2016) Verification of the new grading scale for ocular chronic graft-versus-host disease developed by the German-Austrian-Swiss consensus conference on chronic GVHD. Ann Hematol 95:493-499. https://doi.org/10.1007/ s00277-015-2575-9
31. Kitko CL, Pidala J, Schoemans HM, Lawitschka A, Flowers ME, Cowen EW, Tkaczyk E, Farhadfar N, Jain S, Steven P, Luo ZK, Ogawa Y, Stern M, Yanik GA, Cuvelier GDE, Cheng GS, Holtan SG, Schultz KR, Martin PJ, Lee SJ, Pavletic SZ, Wolff D, Paczesny S, Blazar BR, Sarantopoulos S, Socie G, Greinix H, Cutler C (2021) National Institutes of Health Consensus Development Project on Criteria for Clinical Trials in Chronic Graft-versus-Host Disease: IIa. The 2020 Clinical Implementation and Early Diagnosis Working Group Report. Transplant Cell Ther 27:545-557. https://doi.org/10.1016/j.jtct.2021.03.033

32. Li XM, Hu L, Hu J, Wang W (2007) Investigation of dry eye disease and analysis of the pathogenic factors in patients after cataract surgery. Cornea 26:S16-20. https://doi.org/10.1097/ICO. 0b013e31812f67ca

33. Ram J, Gupta A, Brar G, Kaushik S, Gupta A (2002) Outcomes of phacoemulsification in patients with dry eye. J Cataract Refract Surg 28:1386-1389

34. Choi YJ, Park SY, Jun I, Choi M, Seo KY, Kim EK, Kim TI (2018) Perioperative ocular parameters associated with persistent dry eye symptoms after cataract surgery. Cornea 37:734-739. https://doi.org/10.1097/ICO.0000000000001572

35. Kasetsuwan N, Satitpitakul V, Changul T, Jariyakosol S (2013) Incidence and pattern of dry eye after cataract surgery. PLoS ONE 8:e78657. https://doi.org/10.1371/journal.pone.0078657

36. Saboo US, Amparo F, Shikari H, Dana R (2016) Prevalence of ocular hypertension and glaucoma in patients with chronic ocular graft-versus-host disease. Graefes Arch Clin Exp Ophthalmol 254:923-928. https://doi.org/10.1007/s00417-016-3312-3

37. Labetoulle M, Rousseau A, Baudouin C (2019) Management of dry eye disease to optimize cataract surgery outcomes: two tables for a daily clinical practice. J Fr Ophtalmol 42:907-912. https:// doi.org/10.1016/j.jfo.2019.03.032

38. Chuang J, Shih KC, Chan TC, Wan KH, Jhanji V, Tong L (2017) Preoperative optimization of ocular surface disease before cataract surgery. J Cataract Refract Surg 43:1596-1607. https://doi.org/10. 1016/j.jcrs.2017.10.033

Publisher's Note Springer Nature remains neutral with regard to jurisdictional claims in published maps and institutional affiliations. 